\title{
Electroplasticization of Liquid Crystal Polymer Networks
}

\author{
Hanne M. van der Kooij, Dirk J. Broer, Danqing Liu, and Joris Sprakel*
}

Cite This: ACS Appl. Mater. Interfaces 2020, 12, 19927-19937

Read Online

ACCESS

Wlll Metrics \& More

Article Recommendations

Supporting Information

ABSTRACT: Shape-shifting liquid crystal networks (LCNs) can transform their morphology and properties in response to external stimuli. These active and adaptive polymer materials can have impact in a diversity of fields, including haptic displays, energy harvesting, biomedicine, and soft robotics. Electrically driven transformations in LCN coatings are particularly promising for application in electronic devices, in which electrodes are easily integrated and allow for patterning of the functional response. The morphing of these coatings, which are glassy in the absence of an electric field, relies on a complex
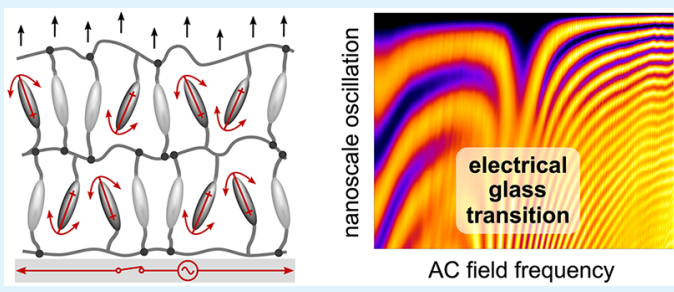
interplay between polymer viscoelasticity, liquid crystal order, and electric field properties. Morphological transformations require the material to undergo a glass transition that plasticizes the polymer sufficiently to enable volumetric and shape changes. Understanding how an alternating current can plasticize very stiff, densely cross-linked networks remains an unresolved challenge. Here, we use a nanoscale strain detection method to elucidate this electric-field-induced devitrification of LCNs. We find how a high-frequency alternating field gives rise to pronounced nanomechanical changes at a critical frequency, which signals the electrical glass transition. Across this transition, collective motion of the liquid crystal molecules causes the network to yield from within, leading to network weakening and subsequent nonlinear expansion. These results unambiguously prove the existence of electroplasticization. Fine-tuning the induced emergence of plasticity will not only enhance the surface functionality but also enable more efficient conversion of electrical energy into mechanical work.

KEYWORDS: liquid crystal polymer networks, glass transition, laser speckle imaging, nonlinear mechanics, nanoscale dynamics, electrical actuation, surface morphing, active materials

\section{INTRODUCTION}

Liquid crystal (LC) polymers have emerged in the past decade as a powerful platform to create active materials with unique functional responses. ${ }^{1}$ These materials synergistically combine the high anisotropy and stimuli-responsiveness of liquid crystals with the tunable viscoelasticity of polymers. The liquid crystal polymer chains are typically cross-linked into a glassy liquid crystal network (LCN) or flexible liquid crystal elastomer (LCE) to impart mechanical strength and shape reversibility while retaining the liquid crystal organization. ${ }^{2,3}$ Triggered by external cues, such as light, ${ }^{4-13}$ heat, ${ }^{14-16}$ humidity, ${ }^{17-19}$ gas, ${ }^{19,20}$ or an electric field, ${ }^{21-27}$ these materials can undergo preprogrammed changes in shape or properties via local adjustment of the liquid crystal order and dynamics. A virtually infinite range of three-dimensional spatiotemporal variations in roughness, motility, wetting, or adhesion can thus be realized. Responsive liquid crystal polymer networks have shown great potential in advanced application fields, including surfaces with adjustable friction for use on robotic fingers, ${ }^{26,28,29}$ on-demand dry self-cleaning coatings for nextgeneration solar cells, ${ }^{25,30,31}$ membranes with tunable permeability for regulated gas transport, ${ }^{16}$ interactively deforming haptic interfaces for Braille displays, ${ }^{32-34}$ and dynamic biointerfaces to guide cell behavior.

Irrespective of the area of application, the common denominator is that functionality arises from a complex competition between solid mechanics on the one hand and the intrinsic desire of liquid crystals to align with external fields on the other. Despite tremendous advances in engineering these "smart" materials, the precise nanomechanics governing shape transformation have remained elusive. Paradoxically, liquid crystal networks are typically glassy and static in the offstate, whereas surface morphing requires the emergence of substantial mobility across different length scales. Topographical deformation must therefore involve field-induced network plasticization.

Increasing the temperature beyond a critical value, denoted as $T_{\mathrm{g}}$, is the most obvious way to cross the glass-to-rubber transition. However, heating is unsuitable for most practical applications and difficult to program in a spatially varying manner to create switchable surface topographies. Inspired by the phenomenon of athermal photofluidization in non-crosslinked azo-polymer films, ${ }^{35-39}$ light-driven LCNs have thus been developed, which locally plasticize upon illumination. The light responsiveness of these materials stems from

Received: January 29, 2020

Accepted: April 8, 2020

Published: April 8, 2020 

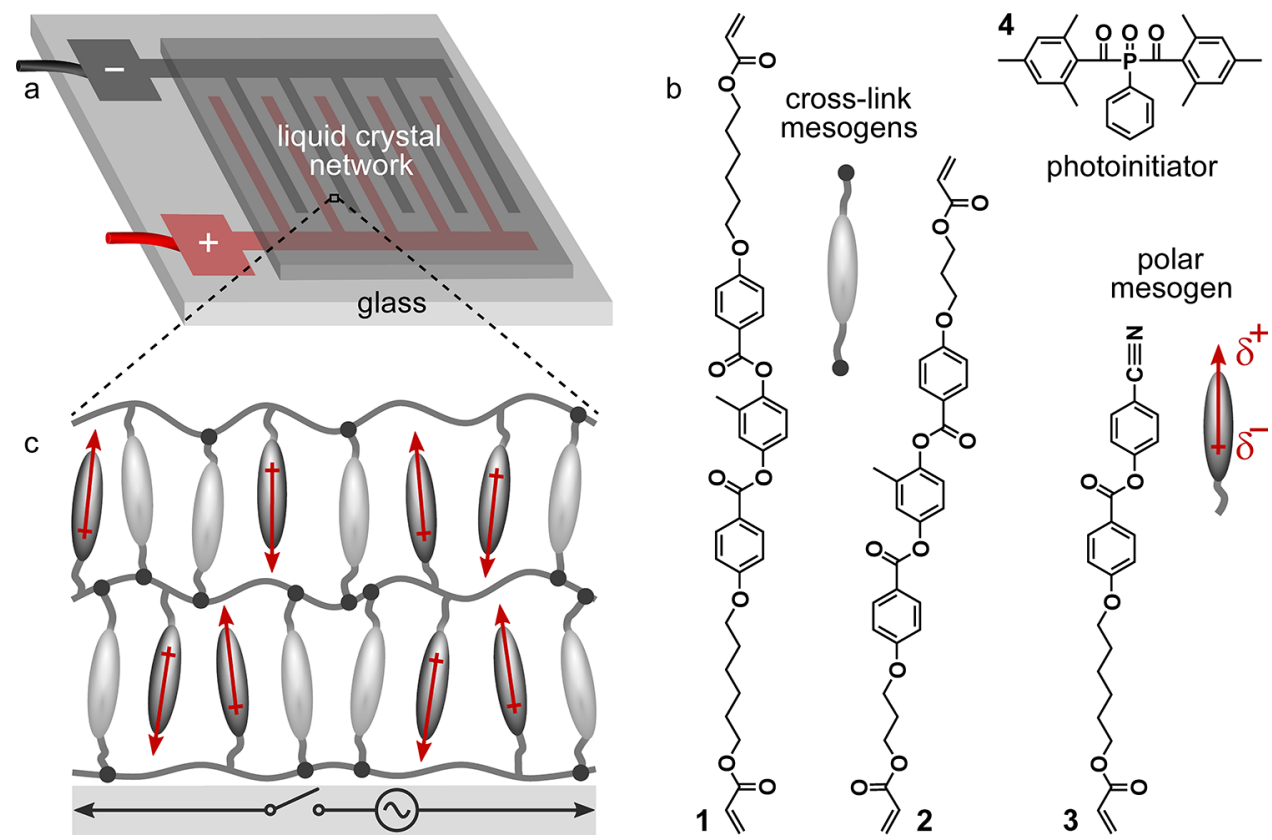

photoinitiator

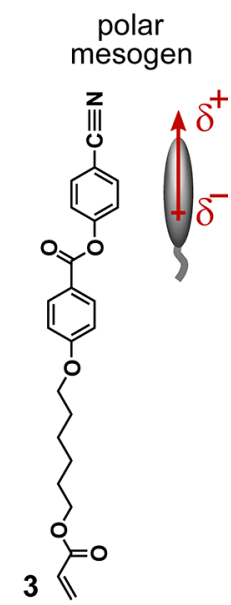

Figure 1. LCN device composition. (a) Schematic of a liquid crystal network coating with embedded interdigitated electrodes, patterned onto a glass support. (b) Chemical structures of the monomers used in the photopolymerization reaction. The two cross-linking mesogens and polar mesogen occur in a 1:1:2 ratio in the network. (c) Schematic zoomed-in cross section of a homeotropically aligned LCN between two electrodes. In the field-on state, the alternating current $(\mathrm{AC})$ field lines are perpendicular to the nematic director. The light gray mesogens are electrically inactive cross links, whereas the dark gray mesogens are permanent dipoles that couple strongly to the field. The dimensions are not to scale.

incorporated azobenzene moieties. Upon UV exposure, azobenzenes isomerize from a rodlike trans form to a bent cis conformation, which is reversed by blue-light illumination. Simultaneous excitation of both the trans and cis isomers via dual-wavelength illumination thus leads to continuous trans $\leftrightarrow$ cis cycling of the azobenzene moieties at a kilohertz rate. ${ }^{40}$ When embedded in an LCN, these oscillatory switching and reorienting azobenzenes exert large internal collective forces on the polymer network, which, in turn, devitrifies the glassy solid. Experimental evidence for the resultant macroscopic network softening was found in a photoinduced decrease in the elastic modulus of up to 2 orders of magnitude. ${ }^{41,42}$ Combined optical and mechanical analysis confirmed the direct correlation between photoisomerization of the azobenzene moieties, photoinduced change in birefringence (i.e., liquid crystal alignment), and photogenerated macroscopic stress. ${ }^{43-45}$ The use of light as a trigger has the advantage of contactless, remote control over the shape-shifting response. Yet photoresponsive polymers also suffer from several drawbacks, such as low light conversion efficiency, thermal instability, and photo-oxidative degradation.

By contrast, electric actuation allows for direct addressing of the material, facile spatial patterning and easy integration into portable devices. ${ }^{24,26}$ Analogous to heat and light, an alternating electric field imposed on mesogens with a large dipole moment can also induce molecular vibrations. These vibrations inside a glassy network are speculated to render the material malleable and dynamic at temperatures far below its $T_{\mathrm{g} .}{ }^{24-26}$ The fact that an electric field can cause mechanical changes and result in pronounced shape-shifting, suggests the existence of a field-induced glass-to-rubber transition. However, conclusive evidence for this "electroplasticization" in liquid crystal polymer networks is lacking. To improve the energy conversion efficiency of electric-field-driven LCNs and minimize dissipation as heat or by plastic deformations, nanoscale insights into the network mechanics at and across this transition are essential. Macroscopic rheology is inherently unsuited to this task, because the electrodes and connected LCN coating are not freestanding but surface constrained. Moreover, the electroplasticization occurs very locally in a spatially heterogeneous manner. While nanoindentation and atomic force microscopy do provide the desired local mechanical information, ${ }^{35,37,46,47}$ these techniques are invasive and limited by their temporal resolution. Clearly, an experimental method is needed that allows dynamic nanomechanical analysis of the network in situ and with high spatiotemporal resolution.

In this paper, we use a recently introduced technique based on laser speckle imaging (LSI) to evidence and study electricfield-induced devitrification of LCNs. This method offers a quantitative analysis of surface strains in these materials and allows in a single experiment to obtain nanomechanical information spanning six decades in time. ${ }^{48,49}$ Laser speckle imaging was originally developed in the medical field for noninvasive imaging of blood flow ${ }^{50-53}$ but has attracted increasing interest of material scientists and proven powerful for probing the nanoscopic mechanics and dynamics of a diversity of complex materials. ${ }^{54-60}$ We recently used LSI to shed more light on the mechanisms underlying shape-shifting of electrically actuated liquid crystal networks. ${ }^{49}$ We revealed how the transformation of surface topography occurs in three distinct hierarchical stages, from the molecular scale upward and from milliseconds to minutes. Here, we extend our previous work by proposing an approach to disentangle elastic and plastic deformations during LCN actuation, providing direct evidence for dissipative processes characteristic of network plasticization. We uncover a complex, nonlinear coupling between the driving electric field and surface dynamics, mediated by induced molecular oscillations. Pronounced changes in the oscillation frequency and plasticity 
occur within a narrow field frequency window, which is dictated by the network nanomechanics. These results not only conclusively evidence the existence of electroplasticization but can also help tailor and optimize the morphological response of these materials.

\section{EXPERIMENTAL SECTION}

Materials. The liquid crystal monomers used in this study are depicted in Figure 1b. LC diacrylates 1 (1,4-bis-[4-\{6-acryloyloxyhexyloxy\}benzoyloxy] benzene, CAS no. 125248-71-7) and 2 (1,4-bis[4-\{6-acryloyloxy-propyloxy\}benzoyloxy]benzene, CAS no. 17406387-7), and cyano-capped LC monoacrylate 3 (4-[4-\{6-[acryloyloxy]hexyloxy\}benzoyloxy] 4-[cyano]benzoate, CAS no. 83847-14-7) are purchased from Merck (U.K.). Photoinitiator 4 (phenylbis[2,4,6trimethylbenzoyl]-phosphine oxide, CAS no. 162881-26-7) is obtained from BASF (Germany). Polyimide 7511L SUNEVER is purchased from Nissan Chemical Corporation (Japan). The reaction solvent dichloromethane is obtained from Sigma-Aldrich. $\mathrm{TiO}_{2}$ (titania) nanoparticles $(\sim 40 \mathrm{~nm}$ diameter, coefficient of variation $\sim 40 \%$, purity $99.5 \%$ ) are purchased from U.S. Research Nanomaterials. All of the components of the LSI setup are obtained from Thorlabs (Germany), unless otherwise specified.

Sample Preparation. $1.5 \times 1.5 \mathrm{~cm}^{2}$ glass substrates are patterned with interdigitated indium tin oxide (ITO) electrodes, $3 \mu \mathrm{m}$ wide with $5 \mu \mathrm{m}$ gaps, covering a total area of $0.5 \times 0.5 \mathrm{~cm}^{2}$. The substrates are cleaned using acetone and isopropanol. To induce spontaneous homeotropic alignment of the mesogens, a $\sim 30 \mathrm{~nm}$ thick film of polyimide $7511 \mathrm{~L}$ is spin-coated onto the glass and baked at $200{ }^{\circ} \mathrm{C}$ for $1 \mathrm{~h}$. Subsequently, the LCN monomers (Figure $1 \mathrm{~b}$ ) are mixed in a ratio of 24.75 wt \% monomer 1, 24.75 wt \% monomer 2, 49.5 wt \% monomer 3, and $1.0 \mathrm{wt} \%$ photoinitiator 4, and dissolved in dichloromethane. The solution is spin-coated onto the baked polyimide film and photopolymerized using UV light from a mercury lamp (OmniCure S2000, Lumen Dynamics Group Inc., Canada) for 5 min under $\mathrm{N}_{2}$ at $26{ }^{\circ} \mathrm{C}$ to lock a nematic orientation. The coating is postcured at $120{ }^{\circ} \mathrm{C}$ under $\mathrm{N}_{2}$ to ensure complete conversion of the monomers. Finally, a thin layer of high-refractive-index $\mathrm{TiO}_{2}$ nanoparticles is deposited onto the surface by spin-coating a $1 \mathrm{wt}$ $\%$ aqueous $\mathrm{TiO}_{2}$ suspension at $2000 \mathrm{rpm}$ for $30 \mathrm{~s}$, to render the coating multiply scattering for LSI. The $\mathrm{TiO}_{2}$ suspension is sonicated for $10 \mathrm{~min}$ prior to spin-coating to disperse any aggregated nanoparticles. The spin-coating conditions are optimized to fulfill the intermediate-scattering requirements, implying that well-developed speckle patterns are obtained (i.e., the scattered photons are randomized), yet the $\mathrm{TiO}_{2}$ coating is sufficiently thin to not alter the surface morphing.

Electric Actuation. An in-plane, sinusoidal electric field is produced by a waveform generator (TGA1241, TTi Inc.) connected to an amplifier (F20A, FLC Electronics, Sweden). Unless otherwise specified, actuation occurs at $25^{\circ} \mathrm{C}$ at a peak-to-peak voltage of $70 \mathrm{~V}$ (i.e., a field strength of $14 \mathrm{~V} / \mu \mathrm{m})$. The output AC signal is monitored using an oscilloscope (TBS1022, Tektronix).

Laser Speckle Imaging. LSI experiments are performed in the backscatter geometry on a custom-built setup described in detail in ref 48. An LSI measurement involves illumination of the area of interest with an expanded coherent laser beam (Cobolt Samba, $1 \mathrm{~W}, \lambda=532$ $\mathrm{nm}$, Cobolt, Sweden) and detection of the multiply scattered photons by a camera. Because the coatings in this study are optically transparent during all of the stages of actuation, the incident photons are predominantly backscattered from $\mathrm{TiO}_{2}$ nanoparticles on the surface. Only multiply scattered photons are detected, as specular and low-order scattering paths are filtered by a linear polarizer perpendicular to the original laser polarization. This results in a socalled speckle pattern on the camera: a spatially random pattern of dark and light "blobs" arising from destructive and constructive interference, respectively. Our camera (Dalsa Genie CCD, CRGM00-H640x, Stemmer Imaging, Netherlands) allows for continuous streaming of these speckle patterns at frame rates up to $200 \mathrm{fps}$.
While the absolute speckle intensity provides information about the surface area, the main power of LSI is its measurement of changes in the speckle intensity over time to gain highly resolved insight into the surface dynamics. The rate of these speckle fluctuations is computed for every pixel using the intensity structure function $d_{2}$

$$
d_{2}(x, y, t, \tau)=\frac{\left\langle[I(x, y, t)-I(x, y, t+\tau)]^{2}\right\rangle}{\langle I(x, y, t)\rangle \cdot\langle I(x, y, t+\tau)\rangle}
$$

where $I$ is the speckle intensity at position $x, y$ and time after switching on or off the field, $t$; and $\tau$ is the time separating two speckle patterns. In this study, the angular brackets denote averaging over a large number of independent speckles, i.e., pixels in space, and contrary to earlier work no time averaging is applied to achieve maximum temporal resolution.

\section{RESULTS AND DISCUSSION}

Liquid Crystal Network Design and Functionality. We study a liquid crystal polymer network whose functional response has been investigated previously. ${ }^{24,49}$ The network is attached to a glass support patterned with interdigitated electrodes and coated with a $\sim 30 \mathrm{~nm}$ polyimide film to ensure homeotropic alignment (Figure 1a). We synthesize the LCN by in situ photopolymerization of a mixture of monomers 1-4 (Figure $1 \mathrm{~b}$ ) in a weight ratio of 1:1:2:0.04. Monomers 1 and 2 are liquid crystal diacrylates that serve as cross-linkers, while additionally their rigid core exhibits thermotropic liquidcrystalline behavior. Monomer 3 is a liquid crystal monoacrylate capped with a cyano group, whose large dielectric anisotropy allows for efficient coupling with an external field. The monomers self-assemble into a nematic phase at room temperature, which is subsequently "frozen" via photopolymerization, initiated by photoinitiator 4. This yields a 2.5-3 $\mu \mathrm{m}$ thick, optically transparent, monodomain liquid crystal network with order parameter of $0.6-0.7 .^{24,49} \mathrm{~A}$ simplified cross section is shown in Figure 1c.

At room temperature in the field-off state, the $\mathrm{LCN}$ is stiff and glassy, with an elastic modulus of $\sim 2 \mathrm{GPa}$ and a thermal glass transition range from 60 to $120{ }^{\circ} \mathrm{C}^{24,49}$ Remarkably, switching on a high-frequency alternating current (AC) rapidly plasticizes the network and has a profound effect on both the individual mesogen dynamics and network mobility as a whole. It is crucial that the field lines are perpendicular to the nematic director so that the dangling polar mesogens experience the maximum dielectric torque and are forced to align with the field. Although this alignment is largely prohibited by the high cross-link density, the dangling dipoles can collectively exert a substantial force on the LCN in which they are embedded, giving rise to yielding of the solid at a critical field strength and frequency. The yield point can be regarded as a stress-induced glass-to-rubber transition, which is common in soft and granular materials. ${ }^{61-63}$ At low stress, the material response is largely elastic, whereas exceeding the yield point involves irreversible plasticity. Yielding and plasticization are typically evoked by an external mechanical perturbation, yet herein the forces are generated from within.

These effects are particularly prominent because the AC field vector continuously changes sign and causes the polar mesogens to pivot in a synchronized, oscillatory manner. By tuning the field frequency, i.e., oscillation frequency, to natural frequencies of the LCN, the pivoting motion not only plasticizes the network but also generates molecular disorder and associated free volume. These dynamic voids decrease the overall network density ${ }^{64}$ and lead to significant surface 

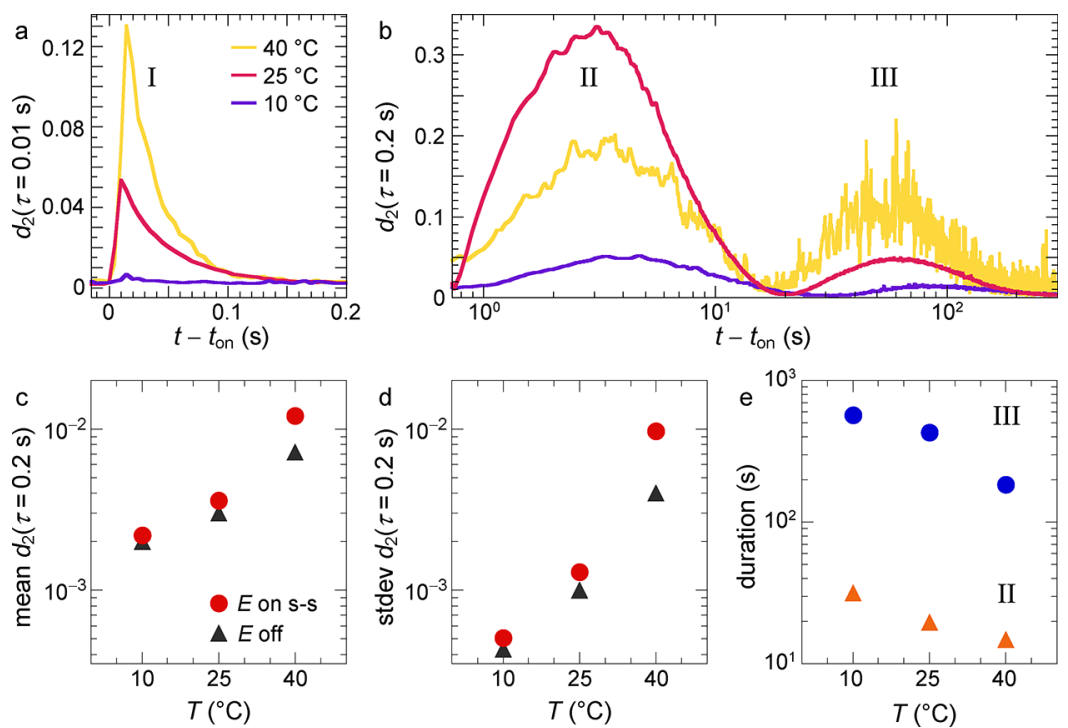

Figure 2. Temperature dependence of electrically driven surface morphing. (a, b) Three distinct stages underlie shape-shifting, as described in detail in our previous work: ${ }^{49}$ (I) fast alignment of the polar mesogens, (II) network plasticization, and (III) gradual deformation. The amplitudes increase monotonically with increasing temperature, except for the electroplasticization stage. (c, d) Nanoscale surface fluctuations in the field-on steady-state (s-s) and field-off state. Both the average motility (c) and its standard deviation (d), i.e., intermittency, rise with increasing temperature. (e) Temperature-dependent kinetics of stages II and III. See Figure S2 for the measurement procedure.

expansion. The possible shape-shifting patterns and dimensions are virtually endless, as the switchable features are not a material property but instead are determined by the electric field configuration. In our device geometry (Figure 1a), the electric field is maximum between electrodes; hence, the fieldinduced expansion manifests as local protrusions. ${ }^{24,49}$

In a previous work, we have used laser speckle imaging to establish a detailed description of the stages leading to surface morphing. ${ }^{49}$ We have shown how a hierarchical chain of events, initiated by an AC field, can ultimately give rise to patterned network expansion and shape-shifting. Molecular cooperativity in space and synchronization in time have proven central in this process, which can be divided into three distinct successive stages: (I) The polar mesogens couple to the alternating field and start to oscillate. (II) Collectively, the mesogens generate large oscillatory stresses, which are the origin of network softening or "electroplasticization." (III) These internal mechanical forces put the network under tension, while simultaneously free volume is formed around the dangling mesogens, jointly leading to network expansion. The resultant height modulation can reach hundreds of nanometers. $^{24-26}$

Local Measurement of Nanodeformations. While previous work has elucidated the nature of the first and last stages, the second and crucial stage in which the glassy network devitrifies has remained elusive to date. In this paper, we focus on unambiguously evidencing the existence of the proposed electric-field-induced glass transition in these coatings. LSI was originally devised for optically thick materials, ${ }^{65}$ yet we previously demonstrated that relevant, semiquantitative results can also be acquired in the intermediate scattering regime, ${ }^{49}$ rendering LSI applicable to thin films coated with highrefractive-index nanoparticles as used in this study. We thus spin-coat a thin layer of $\mathrm{TiO}_{2}$ pigments onto the LCN surface and illuminate the center with a coherent, collimated laser beam. The laser photons are scattered several times inside the $\mathrm{TiO}_{2}$ layer before traveling back and reaching the camera detector. Although we operate in the limit of weak multiple scattering because of the thin-film geometry, we filter photons that have undergone no or too few scattering events by a linear polarizer in front of the camera. All of the remaining photons have traveled different path lengths and have thus accumulated phase shifts, resulting in a random interference pattern on the camera called the speckle pattern. Changes in this pattern reflect displacements of the $\mathrm{TiO}_{2}$ nanoparticles, which, in turn, must ensue from deformations of the network surface because the pigments are firmly attached.

The characteristic timescale of speckle intensity fluctuations is directly related to the rate of surface deformations, and the fluctuation amplitude is related to the amount of deformation. Surface strains of the order of nanometers are detectable, as cumulative nanometric changes in scattering events rapidly add up to measurable variations in the speckle pattern. We quantify the surface motion using the structure function $d_{2}$ (see the Experimental Section). $d_{2}(x, y, t, \tau)$ is a four-dimensional function of spatial coordinates $x$ and $y$; time after switching the field on or off, $t$; and lag time between correlated speckle patterns, $\tau$. Computing $d_{2}$ as a function of $\tau$ allows us to spectrally resolve processes occurring on different timescales. ${ }^{48,57,58}$ For example, stage I is very fast and thus requires analysis at short $\tau=0.01 \mathrm{~s}$ to prevent loss of temporal resolution (Figure 2a), whereas stages II-III benefit from analysis at longer $\tau=0.2 \mathrm{~s}$ to obtain sufficient speckle decorrelation (Figure $2 \mathrm{~b}$ ). We previously used the imaging power of LSI to create spatial representations of the dynamics. $^{49}$ In this paper, we average over a small area $\left(0.040 \mathrm{~mm}^{2}\right)$ to further enhance the signal-to-noise ratio.

Temperature Dependence of Morphing Stages. We first focus on unraveling the combined effects of heat and electricity, both of which enhance molecular vibrations. However, while heat causes random reorientation of the mesogens and a concomitant decrease in molecular order, ${ }^{23,35}$ an AC field involves anisotropic angular displacement of the mesogens. ${ }^{22}$ In fact, switching on a $900 \mathrm{kHz}$ field even causes some in-plane birefringence to build up, due to the biased reorientation of mesogens along the field lines. The change of 

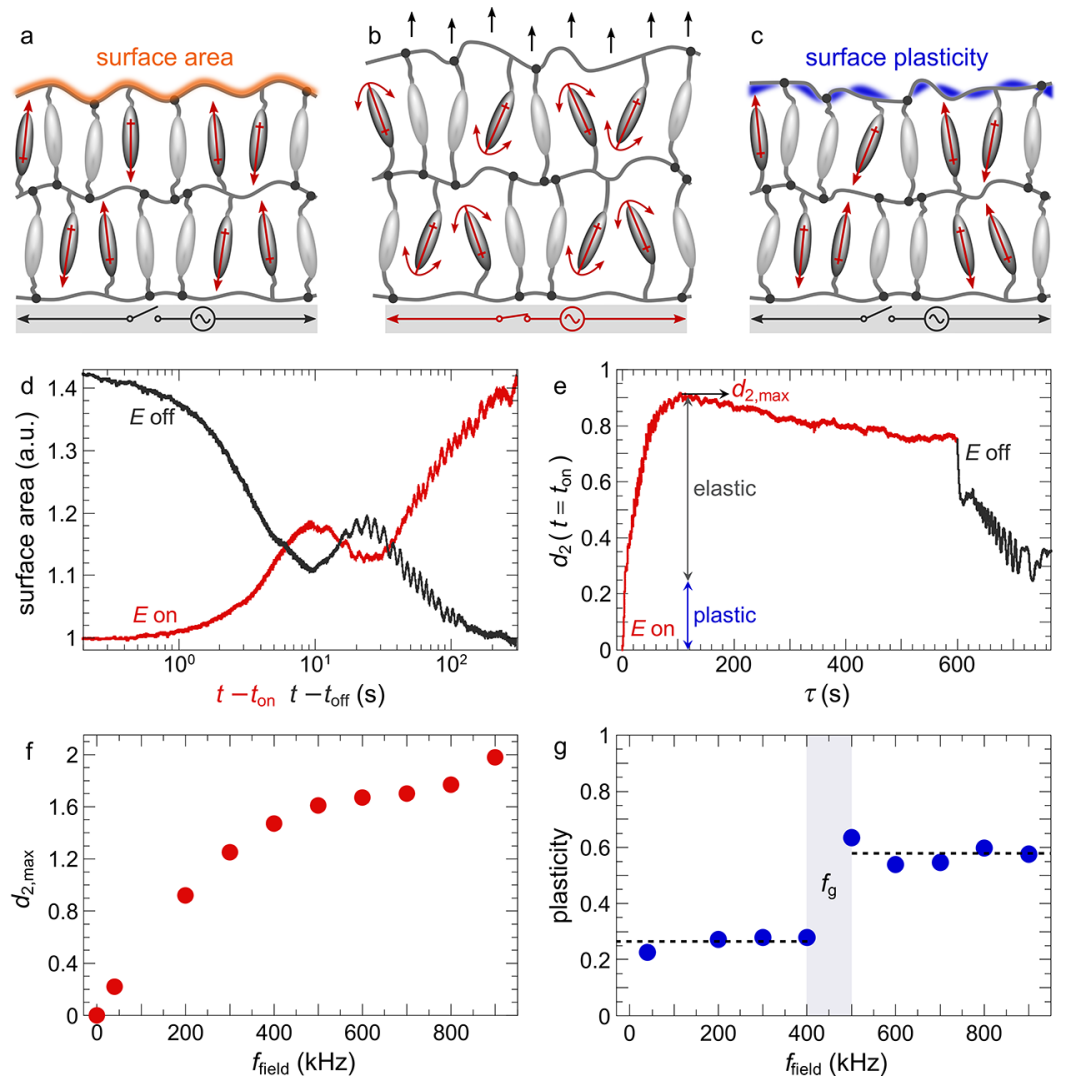

Figure 3. Shape-shifting elasticity and plasticity. $(a-c)$ Schematic cross sections of a liquid crystal polymer network in the field-off state (a), after switching on a high-frequency AC field (b), and after field switch-off and relaxation (c). The electric field drives the polar mesogens into oscillation, causing local network plasticization and expansion. After field switch-off, elastic displacements are restored yet plastic deformations (in blue) remain. The dimensions are not to scale. (d) Change in $\tilde{A}$ after switching a $200 \mathrm{kHz}$ AC field on (red line) and off (black line), which are fully symmetric. (e) The corresponding surface structure function, referenced to $t=t_{\mathrm{on}}$, shows hysteresis between field switch-on (red line) and -off (black line). The drop in $d_{2}$ measures the reversible, elastic deformation, whereas the plateau at long $\tau$ measures plastic strains. (f) Maximum $d_{2}$, reflecting total elastic plus plastic deformation, versus field frequency. $(\mathrm{g})$ Unrecovered fraction of the surface structure change. A sharp transition from 400 to $500 \mathrm{kHz}$ signals a sudden rise in plasticity, corresponding to a field-induced glass transition around the critical frequency $f_{\mathrm{g}}$.

birefringence is, however, only $\sim 0.03$, implying that the mesogens vibrate with small amplitude and that their orientational redistribution upon actuation is limited.

To test if the field-induced directional changes dominate over the heat-induced loss of order, we measure the dielectric response (stage I) at three temperatures. Our samples are thermostated to the desired temperature well before electric induction. We quantify the local, nanoscale surface deformation using the above described $d_{2}$ structure function. Molecular displacements inside the liquid crystal network, such as tilting of the polar mesogens, will cause a small but detectable deformation of the surface. $d_{2}$ reflects the magnitude of these surface strains and thus indirectly measures the degree of internal network perturbations.

In an earlier work, we found that the deformation kinetics during the dielectric response are strikingly universal for our class of LCNs. ${ }^{49}$ This observation is confirmed at different temperatures: $d_{2}$ peaks within $10-15 \mathrm{~ms}$ after field switch-on as the field torques the polar mesogens, followed by exponential relaxation of the network over $\sim 200 \mathrm{~ms}$ (Figure 2a). Conversely, the amount of surface deformation depends strongly on the mesogen-field interactions. We previously uncovered a distinct positive relationship between the deformation amplitude and field frequency, voltage, and angle between the molecular dipoles and field lines. ${ }^{49}$ At elevated temperature, the network permittivity and dielectric interactions are larger; ${ }^{24}$ this indeed enhances the mesogen alignment and consequent LSI signal (Figure 2a). Moreover, the network weakens with increasing temperature, ${ }^{49}$ allowing the polar mesogens to pivot with greater amplitude. Clearly, these anisotropic phenomena far outweigh the randomizing effects of heat.

Also the final height modulation in stage III increases with rising temperature, thanks to the larger network mobility and permittivity (Figure $2 \mathrm{~b}$ ). Note that the surface temperature remains below the polymer $T_{\mathrm{g}}\left(60-120{ }^{\circ} \mathrm{C}\right)$ in all of the experiments, since dielectric heating is only $\sim 10{ }^{\circ} \mathrm{C}$, as measured using an infrared sensor. ${ }^{24,49}$ This limited temperature rise is one of the key differences between electroplasticization and the well-established process of highfrequency welding (see the Supporting Information for details). ${ }^{6,67}$ Temperature gradients in the coating are negligible, thanks to its small thickness $(2.5-3 \mu \mathrm{m})$ and attachment to a relatively thick glass substrate $(1 \mathrm{~mm})$, which dissipates heat from the LCN by acting as a heat sink.

Qualitatively, stages I-III are highly similar for the three temperatures, confirming how nanoscale motions are amplified in time and space in a stepwise manner (Figure 2a,b). Even for a different (cholesteric ${ }^{68}$ ) alignment of identical chemical composition, we find the same three stages (Figure S1). Nevertheless, quantitative comparison reveals important differences. First evidence for electroplasticization is found in stage 

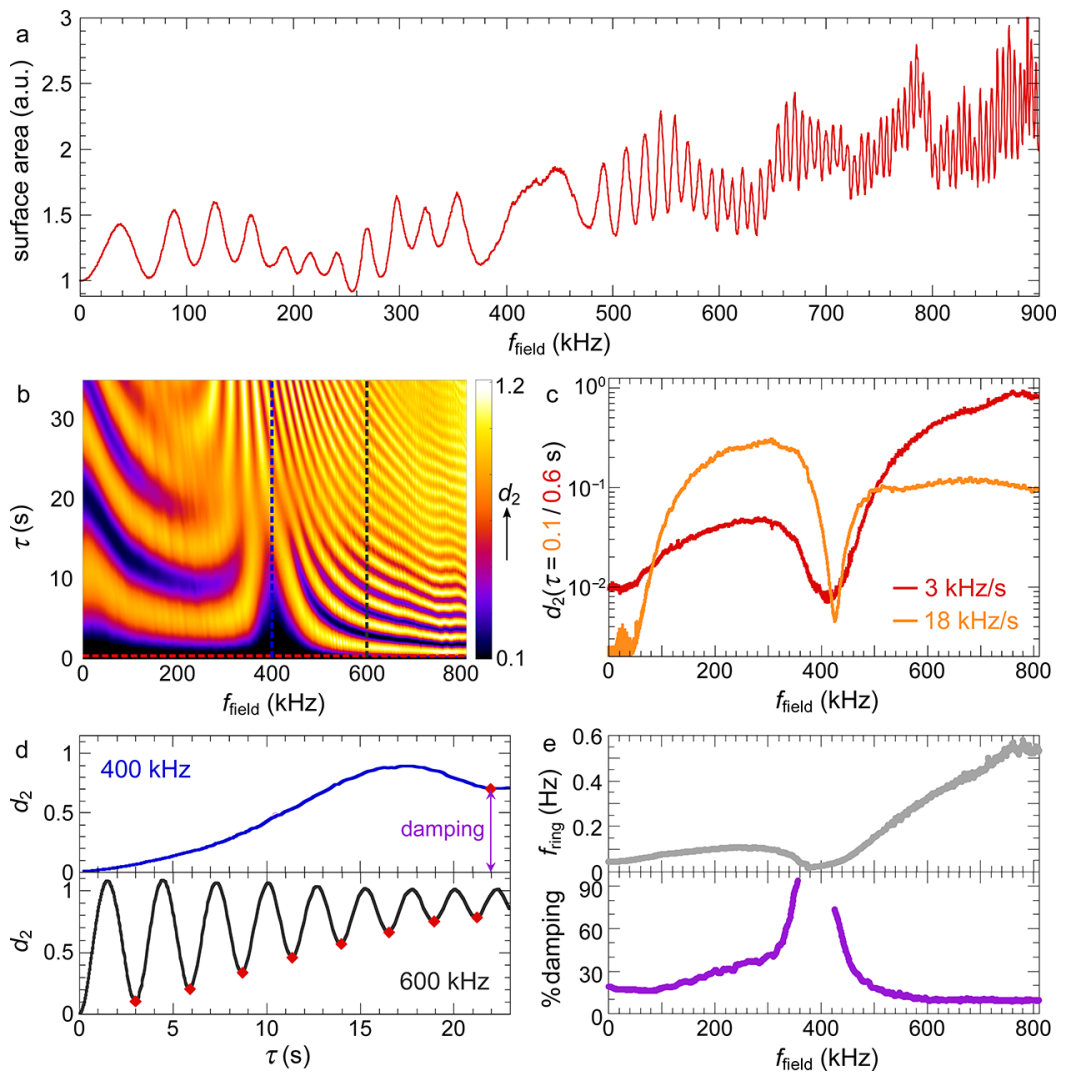

Figure 4. Electroplasticization by high-frequency AC field. (a) Change in $\tilde{A}$ and (b) $d_{2}$ spectrum during a field frequency sweep at $3 \mathrm{kHz} / \mathrm{s}$. The surface expands with increasing $f_{\text {field }}$ and exhibits undulations with decreasing period. A pronounced divergence of the period around critical frequency $f_{\mathrm{g}}=400 \mathrm{kHz}$ coincides with (c) a drop in the surface motility, signaling energy dissipation characteristic of a glass transition. The colors indicate different sweep rates. Note that a 6-fold higher $\tau$ is used to compute $d_{2}$ for the 6-fold slower sweep. (d) Vertical cross sections through the spectrum in (b) at 400 and $600 \mathrm{kHz}$. Distinct elastic ringing is evident at $600 \mathrm{kHz}$ : the surface does not expand monotonically with increasing lag time $\tau$ but recurrently returns to an earlier position, manifest as a train of "echoes" marked by red diamonds. (e) Field frequency dependence of the ringing frequency (top) and percent damping per period (bottom), extracted from the $d_{2}(\tau)$ curves. Both show a cross-over at $400 \mathrm{kHz}$. While the ringing frequency vanishes at $f_{\mathrm{g}}$, dissipation-induced damping diverges.

II, whose amplitude does not increase monotonically with increasing $T$ analogous to the other stages but instead is smaller at $40{ }^{\circ} \mathrm{C}$ than at $25{ }^{\circ} \mathrm{C}$. This observation is perfectly in line with the fact that closer to the network $T_{\mathrm{g}}$, less electrical plasticization is required to yield the solid matrix, even though the subsequent expansion is larger. Other effects of the temperature are relatively modest yet can still be well identified using LSI. For example, the mean surface motility in the fieldoff state (black triangles) and in the field-on steady state (red circles, $500 \mathrm{~s}$ after field switch-on) both show a clear positive dependence on temperature (Figure 2c). The difference between 10 and $40{ }^{\circ} \mathrm{C}$ remains however within an order of magnitude. Equivalently, the standard deviation of $d_{2}$, a measure for the intermittency of the surface fluctuations, also grows with increasing temperature (Figure 2d). This intermittency is clearly visible in the $40{ }^{\circ} \mathrm{C}$ curve in Figure $2 \mathrm{~b}$ and indicates that a softer network responds more chaotically to the mesogen oscillations. These surface fluctuations are, e.g., beneficial for dirt removal applications, ${ }^{25}$ favoring operation of this device at elevated temperature, in addition to achieving larger height modulations. Moreover, heat accelerates the kinetics of plasticization and expansion, as evident from a slight decrease in the duration of stages II and III (Figures 2e and S2).

Disentangling Elastic and Plastic Strains. We have shown how a $900 \mathrm{kHz}$ AC field activates three well-defined hierarchical dynamic stages over a range of temperatures. However, at each temperature, a critical field frequency exists below which no significant surface morphing occurs. According to digital holography microscopy (DHM) measurements, the desired functionality of the LCN emerges only at field frequencies $\left(f_{\text {field }}\right)$ of $500 \mathrm{kHz}$ and above (see Figure $3 \mathrm{~d}$ in ref 24). Efficient plasticization is crucial in this regard. At low $f_{\text {field }}$ the surface deformation is largely elastic and reversible, limited by the glassy nature of the network. At sufficiently high field frequencies, the surface expansion becomes nonlinear, with concomitant irreversible rearrangements inside the coating. During such a plastic response, not only is energy dissipated but the material also adopts a new shape, as depicted schematically in Figure $3 \mathrm{a}-\mathrm{c}$. The network microstructure will change nonaffinely to accommodate for these plastic strains. To shed more light on the phenomenon of electroplasticization, distinguishing local elastic from plastic strains is key. LSI is a highly sensitive, versatile, and effective means to identify elastic-plastic transitions in LCNs.

From a single LSI experiment, we can not only extract the surface motility $d_{2}$, which quantifies the changes in scattered intensity, but also the surface area, which relates to the absolute scattered intensity. More precisely, the average speckle intensity $\langle I\rangle$ is proportional to the surface concentration of nanoparticles $c_{\mathrm{s}}=n / A$, with $A$ the surface area (defined in Figure $3 a$ ). We can thus compute the reduced 
change in surface area over time: $\tilde{A}(t) \equiv A(t) / A_{0}=I_{0} / I(t)$. Interestingly, at low field frequency $(200 \mathrm{kHz})$, the increase in $\tilde{A}$ upon field switch-on is exactly reversed upon field switch-off and displays remarkably little hysteresis (Figure 3d). By contrast, the surface structure will not fully recover to its initial value after an on-off cycle and will exhibit plastic rearrangements. These rearrangements do not alter the overall shape of the coating nor the surface roughness but manifest themselves as irregular nanoscale changes of the surface, which are fixed when the coating resolidifies (see Figure $3 \mathrm{c}$ in blue).

To quantify this plasticity, we focus on the entire speckle pattern, i.e., the structure function $d_{2}$ rather than on the average speckle intensity. We start from the same reference point as in Figure 3d, which is the last speckle pattern before induction at $t=t_{\mathrm{on}}$. As the network expands over time, the surface structure and corresponding speckle pattern will change gradually and incrementally with respect to the reference pattern, leading to a progressive rise of $d_{2}$. The lag time $\tau$ here equals the time after electric induction. Switching off the field at $t-t_{\mathrm{on}}=600 \mathrm{~s}$ allows the surface to slowly return to its original shape, thus causing a decrease in $d_{2}$ as the shape recovery is largely elastic. Remarkably, while the change in surface area is symmetric between field switch-on and -off (Figure 3d), the surface structure does not follow the same route upon expansion and contraction (Figure 3e). This hysteresis is clearly apparent from the finite $d_{2}$ plateau at long $\tau$, which reflects plastic rearrangements. In a perfectly elastic system, each surface-attached $\mathrm{TiO}_{2}$ nanoparticle would return exactly to its initial position after shape recovery, since the nanoparticles adhere strongly to the network and display negligible lateral displacement; hence, the original unperturbed speckle pattern would be regained, with $d_{2} \rightarrow 0$. The height of the plateau at long $\tau$ is thus a measure for the fraction of the surface undergoing irreversible deformation. At $200 \mathrm{kHz}$, this height is approximately a quarter of the maximum $d_{2}\left(d_{2, \max }\right.$ Figure 3e).

Although the plasticity is relatively small at low field frequency, we must take into account that the total surface expansion is also rather limited. Digital holography even fails to detect any deformation, ${ }^{24}$ implying that the height modulation is less than $10 \mathrm{~nm}$. This is confirmed by the relatively small $d_{2, \max }$, which equals 0.9 instead of the intrinsic maximum value of 2 . Indeed, the maximum $d_{2}$ is a good gauge of the total deformation amplitude; in agreement with DHM and simulations, ${ }^{24}$ we find that $d_{2, \max }$ rises rapidly with increasing $f_{\text {field }}$ at low field frequencies and reaches an optimum around $900 \mathrm{kHz}$ (Figure 3f). The reduced change in surface area $\tilde{A}$ displays a very similar trend (Figure S3a). By contrast, the plasticity has a strikingly different dependence on $f_{\text {field }}$, showing two regimes with an abrupt transition around $400-500 \mathrm{kHz}$ (Figures $3 \mathrm{~g}$ and $\mathrm{S} 3 \mathrm{~b}$ ). We have estimated this plasticity from the ratio between the long- $\tau$ plateau $\left(d_{2, \min }\right)$ and $d_{2, \max }$. Although this approach does not provide absolute values for the amount of plasticity, it offers a means to compare the relative plasticity with high resolution. Clearly, surface morphing occurs for all of the field frequencies with only partial recovery (Figure $3 \mathrm{~g}$ ). At low $f_{\text {field }}$ this deformation is small and largely in the linear elastic region, i.e., the field induces few irreversible structural changes. Beyond a critical frequency of $400-500 \mathrm{kHz}$, there is a sharp cross-over toward significantly enhanced local plasticization. Analogous to the thermal glass transition $\left(T_{\mathrm{g}}\right)$ of the $\mathrm{LCN}$, this critical frequency may signal the electrical glass transition $f_{\mathrm{g}}$.
Electroplasticization by High AC Frequency. If the critical field frequency is truly an electrical glass transition, we expect a peak in energy dissipation around $f_{\mathrm{g}}$ that accompanies the yielding process. To investigate this, we subject the LCN to a linear field sweep from 0 to $900 \mathrm{kHz}$ at $3 \mathrm{kHz} / \mathrm{s}$. Interestingly, the surface does not expand linearly with increasing field frequency but instead exhibits pronounced undulations, as the dielectric interactions between the field and LCN keep changing during the sweep (Figure 4a). This oscillatory motion is reminiscent of elastic ringing. The "ringing" of this coating is a complex phenomenon: not only does the field frequency change over time but also the network modulus and density fluctuate strongly in response to the field, thus causing recoil and overshoot of the surface as it fails to meet the rapidly changing resonance conditions. We previously uncovered that the ringing frequency is proportional to the field frequency when a constant $f_{\text {field }}$ is applied. ${ }^{49}$ A similar trend is visible during this sweep, although a discontinuity appears between 400 and $500 \mathrm{kHz}$ (Figure 4a).

To gain more insight into the origin of this discontinuity, we again revert to the structure function $d_{2}$, which we compute as a function of both $f_{\text {field }}$ and $\tau$ (Figure $4 \mathrm{~b}$ ). A striking array of periodic undulations emerges, confirming that the surface repeatedly expands and contracts to an earlier position in an oscillatory manner. However, the oscillation frequency does not increase monotonically but instead diverges around 400 $\mathrm{kHz}$, suggesting a decoupling of the field and network expansion. This critical transition, also observed in Figure 3g, recurs in the one-point $d_{2}$ (Figure $4 c$, red line), which is essentially a horizontal cross section through Figure $4 \mathrm{~b}$. It is likely not governed by kinetics but predominantly thermodynamic in origin, since the cross-over occurs after $>100 \mathrm{~s}$ of sweeping the field, much longer than typical dielectric and relaxation timescales of the material. Moreover, a highly similar transition is visible for a faster $f_{\text {field }}$ sweep of $18 \mathrm{kHz} / \mathrm{s}$ (Figure $4 c$, orange line). For a very low sweep rate, the response is somewhat different, as elaborated in Figure S4.

Nanomechanical Insight from Surface Oscillations. The periodicity of the $d_{2}(\tau)$ functions offers an opportunity to extract nanomechanical information from the ringing frequency and damping. While a typical $d_{2}$ curve increases continuously with increasing $\tau$, we here observe a train of echoes at integral multiples of the oscillation period. These echoes are particularly well visible when taking vertical cross sections through Figure $4 \mathrm{~b}$, as shown for $f_{\text {field }}=400$ and 600 $\mathrm{kHz}$ in Figure $4 \mathrm{~d}$. At $400 \mathrm{kHz}$, the ringing frequency $f_{\text {ring }}$ is very low, yet at $600 \mathrm{kHz}, d_{2}(\tau)$ displays nearly perfect sinusoidal undulation. The amplitude of the echoes (red diamonds) gradually diminishes with increasing $\tau$ because each oscillation involves additional rearrangements. Note that the speckle fluctuations are averaged only over space, without any time averaging or smoothing, highlighting the remarkable sensitivity of LSI.

For each field frequency, we determine the ringing frequency from the position of the first $d_{2}(\tau)$ maximum. In line with our earlier observations, we do not find a linear relationship between $f_{\text {ring }}$ and $f_{\text {field }}$ but rather a highly nonmonotonic dependence (Figure 4e, top). The discontinuity around 400 $\mathrm{kHz}$ signifies a transient rise in energy dissipation indicative of a glass transition, with electrical energy converted into molecular rearrangements instead of powering surface undulations. Fourier transform of the raw intensity signal yields an identical trend (Figure S5), verifying the robustness 

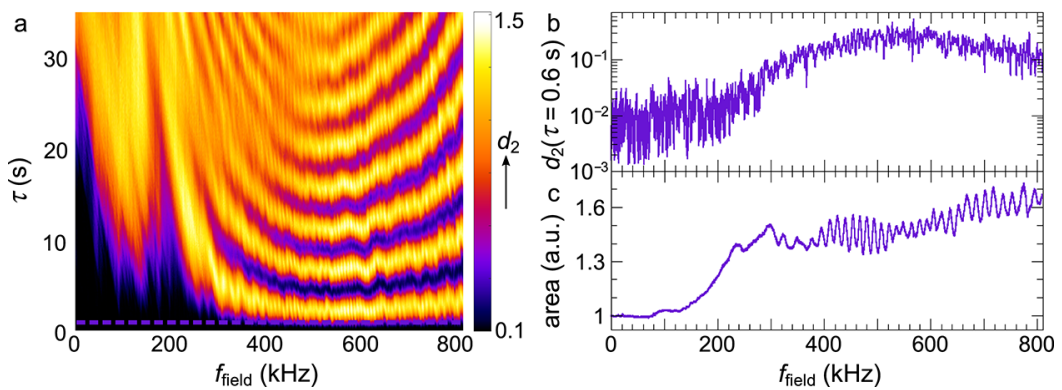

Figure 5. Thermal shift of $f_{\mathrm{g}}$. (a) $d_{2}$ spectrum, (b) surface motility, and (c) change in $\tilde{A}$ during a field frequency sweep at $3 \mathrm{kHz} / \mathrm{s}$ and $T=40^{\circ} \mathrm{C}$. The discontinuity around $\sim 200 \mathrm{kHz}$ indicates an earlier onset of electroplasticization than at $25{ }^{\circ} \mathrm{C}$. Note also the considerably reduced undulation frequency in (a) compared with Figure $4 \mathrm{~b}$.

of our method. Further validation is obtained when comparing the amount of damping at different field frequencies (Figure 4d): while at $400 \mathrm{kHz}$ the oscillation is almost fully damped after a single period, at $600 \mathrm{kHz}$, the damping per period is only $9 \%$. We estimate the percent damping as the ratio between the first $d_{2}$ minimum (i.e., echo) and maximum. Plotted versus $f_{\text {field }}$, this again reveals two distinct regimes centered at $400 \mathrm{kHz}$ (Figure 4e, bottom). The percent damping can be considered analogous to the loss tangent $\tan (\delta)$ used in macroscopic mechanical analysis, a measure for the relative importance of dissipative and elastic processes. Indeed, a peak in $\tan (\delta)$ is a signature of thermal glass transition. $^{41}$

Thermal Shift of Electrical Glass Transition. The final support for our hypothesis is found in the temperature dependence of $f_{\mathrm{g}}$. Thermally and electrically induced plasticization are closely connected, as already found in Figure $2 \mathrm{~b}$, stage II. Specifically, heat is expected to lower the field frequency at which electroplasticization occurs. We thus measure the surface deformation at $T=40{ }^{\circ} \mathrm{C}$, which indeed displays a sharp discontinuity at a reduced $f_{\text {field }}$ of $\sim 200 \mathrm{kHz}$ (Figure 5a). Taking a horizontal cross section through the spectrum at $\tau=0.6 \mathrm{~s}$, equivalent to Figure $4 \mathrm{c}$, confirms the downward shift of $f_{\mathrm{g}}$ (Figure $5 \mathrm{~b}$ ). Interestingly, also the $d_{2}$ regime at low $f_{\text {field }}$ is shifted toward longer timescales and consequently the short- $\tau d_{2}$ curve does not show two clear regimes. A likely explanation is a decreased amount and frequency of elastic deformations compared with viscous dissipation. Note that the $d_{2}$ intermittency is again much stronger than that at $25{ }^{\circ} \mathrm{C}$ (compare Figure $5 \mathrm{~b}$ with Figure $4 c$ ), indicating a more chaotic response of the $\mathrm{LCN}$ to the field, characteristic of the higher thermal energy. Also the change in surface area $\tilde{A}$ during the field sweep corroborates the faster onset of electroplasticization: while at $25{ }^{\circ} \mathrm{C}$, significant surface expansion starts only around $400 \mathrm{kHz}$ (Figure 4a), at $40{ }^{\circ} \mathrm{C}$, a rapid rise in $\tilde{A}$ is manifest already at $200 \mathrm{kHz}$ (Figure 5c).

Microscopic Interpretation. Our results reveal the existence of a true electric-field-induced devitrification of the LC polymer network at a critical field frequency of several hundreds of kilohertz. This raises the question what microscopic mechanisms inside the material underlie this glass transition.

The alternating electric field induces a pivoting motion of the dangling mesogens. Since these molecular oscillations are constrained and frustrated by the rigid environment of the glassy and cross-linked network, it is likely that they exhibit substantial disorder, which induces nonaffinity in the thermal motions. In other rigid soft materials, both ordered ${ }^{69}$ and glassy, $^{70,71}$ it was shown that increasing the amplitude of thermal nonaffinities leads to a loss of material rigidity and possibly a solid-to-liquid transition, akin to the one we observe here. Moreover, the dynamic disorder created by the fieldinduced oscillations inside the LCN causes a volumetric expansion, ${ }^{24,40,64}$ which reduces the material density and increases the effective distances between mesogens. This necessarily reduces the strength of the van der Waals interactions between neighboring mesogens and provides an additional mechanism for the rigidity loss that must accompany devitrification. Rigidity in our LCN derives from both covalent bonds between monomers in the network and supramolecular interactions between mesogens, probably of the van der Waals type. The covalent network is most likely unaffected by the actuation, since the observed shape and volumetric changes are fully reversible. ${ }^{24}$ The electroplasticization must therefore relate to a reduction of the supramolecular van der Waals interactions.

Finally, the question remains why devitrification in our experiments occurs at frequencies of hundreds of kilohertz. These frequencies are far too small to reflect natural dipolar resonances at the molecular level. By contrast, resonatorrelated vibrations that link to sample dimensions, such as the electrode distances, are much slower. Rather, these frequencies probably reflect the excitation of collective modes of motion of many mesogens in tandem, analogous to the excitation of phonons in an ordered lattice. We thus postulate that the critical field frequency to cross the glass transition is governed by long-ranged molecular vibrations related to intermolecular interactions. At present, there is insufficient knowledge of the vibrational spectrum of collective modes in liquid crystal materials to provide a clear explanation for the values we have found. Since it appears that these collective effects are crucial to explain plasticization and actuation of active LCNs at a microscopic level, theoretical efforts in this area are highly needed.

\section{CONCLUSIONS}

Research into the engineering of active LCNs to date has relied on the fundamental premise that oscillating molecular dipoles cause plasticization of the network, ${ }^{24-26,49}$ which ultimately enables the shape-shifting response from which these materials derive their appeal. We here provide the first conclusive evidence for this hypothesis, by revealing how LCN coatings are "electroplasticized" by a high-frequency AC field. Using a nanoscale strain detection method based on laser speckle imaging, we uncover how small oscillatory forces, generated by 
embedded responsive mesogens, collectively induce an athermal glass transition. Molecular synchronization is crucial in this regard, as plasticity emerges only beyond a critical field frequency of $400-500 \mathrm{kHz}$. Around this frequency, we find pronounced discontinuities in strain nonlinearity, oscillation frequency, and damping. These phenomena have interesting analogies with mechanical yielding processes observed in a variety of soft materials, ranging from yield stress fluids ${ }^{61,72}$ to polymeric solids $^{62,73}$ and granular media, ${ }^{63,74}$ where mechanical stress can induce a solid-to-liquid transition. In this study, the mechanical disturbance underlying the field-induced glass transition is not applied from the outside but generated from within by the frustrated motion of polar mesogens in response to an electric field.

Our results not only support the existence of an electrical glass transition but also provide new insights into optimization of the surface functionality. For example, fine-tuning the oscillation frequency to match human tactile perception allows for more efficient dynamic Braille interfaces. ${ }^{32-34}$ In the context of biointerfaces, the spatiotemporal nanomechanics are key in signaling and regulating cell behavior. ${ }^{10,23}$ Most practical applications will benefit from reduced field frequencies, which involve less complex device configurations and lower power consumption. On the basis of the measured equivalence between $T$ and $f_{\text {field }}$, we thus recommend lowering the network $T_{g}$, e.g., by extending the alkyl spacer length or by decreasing the cross-linking density.

\section{ASSOCIATED CONTENT}

\section{SI Supporting Information}

The Supporting Information is available free of charge at https://pubs.acs.org/doi/10.1021/acsami.0c01748.

Differences between electroplasticization and highfrequency welding, morphing stages for a cholesteric alignment, temperature-dependent duration of stage II, an extension of Figure 3, surface motility during a field frequency sweep at $0.9 \mathrm{kHz} / \mathrm{s}$, and field frequency dependence of the ringing frequency by Fourier transform (PDF)

\section{AUTHOR INFORMATION}

\section{Corresponding Author}

Joris Sprakel - Physical Chemistry and Soft Matter, Wageningen University \& Research, 6708 WE Wageningen, The Netherlands; 10 orcid.org/0000-0001-6532-4514; Email: joris.sprakel@wur.nl

\section{Authors}

Hanne M. van der Kooij - Physical Chemistry and Soft Matter, Wageningen University \& Research, 6708 WE Wageningen, The Netherlands; Dutch Polymer Institute (DPI), 5600 AX

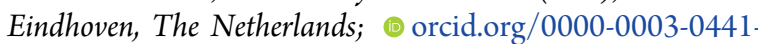
1109

Dirk J. Broer - Stimuli-Responsive Functional Materials and Devices, Department of Chemical Engineering and Chemistry and Institute for Complex Molecular Systems, Eindhoven University of Technology, 5612 AE Eindhoven, The Netherlands

Danqing Liu - Stimuli-Responsive Functional Materials and Devices, Department of Chemical Engineering and Chemistry and Institute for Complex Molecular Systems, Eindhoven University of Technology, 5612 AE Eindhoven, The

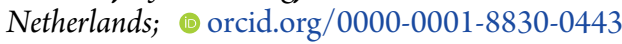

Complete contact information is available at:

https://pubs.acs.org/10.1021/acsami.0c01748

\section{Notes}

The authors declare no competing financial interest.

\section{ACKNOWLEDGMENTS}

The research of H.M.v.d.K. forms part of the research programme of the Dutch Polymer Institute (DPI), project \#781. The contribution of D.J.B. was supported by the European Research Council with the ERC Advanced Grant 66999 (VIBRATE). The work of D.L. is part of the NWO VENI research programme with project number 15135 and that of J.S. of the NWO VIDI research programme with project number 723.016.001. Merck is acknowledged for providing ITO IDE.

\section{REFERENCES}

(1) White, T. J.; Broer, D. J. Programmable and Adaptive Mechanics with Liquid Crystal Polymer Networks and Elastomers. Nat. Mater. 2015, 14, 1087-1098.

(2) Ge, F.; Zhao, Y. Microstructured Actuation of Liquid Crystal Polymer Networks. Adv. Funct. Mater. 2019, No. 1901890.

(3) Ware, T. H.; McConney, M. E.; Wie, J. J.; Tondiglia, V. P.; White, T. J. Voxelated Liquid Crystal Elastomers. Science 2015, 347, 982-984.

(4) Finkelmann, H.; Nishikawa, E.; Pereira, G. G.; Warner, M. A New Opto-Mechanical Effect in Solids. Phys. Rev. Lett. 2001, 87, No. 015501.

(5) Yu, Y.; Nakano, M.; Ikeda, T. Photomechanics: Directed Bending of a Polymer Film by Light. Nature 2003, 425, 145.

(6) Camacho-Lopez, M.; Finkelmann, H.; Palffy-Muhoray, P.; Shelley, M. Fast Liquid-Crystal Elastomer Swims into the Dark. Nat. Mater. 2004, 3, 307-310.

(7) McConney, M. E.; Martinez, A.; Tondiglia, V. P.; Lee, K. M.; Langley, D.; Smalyukh, I. I.; White, T. J. Topography from Topology: Photoinduced Surface Features Generated in Liquid Crystal Polymer Networks. Adv. Mater. 2013, 25, 5880-5885.

(8) Iamsaard, S.; Aßhoff, S. J.; Matt, B.; Kudernac, T.; Cornelissen, J. J. L. M.; Fletcher, S. P.; Katsonis, N. Conversion of Light into Macroscopic Helical Motion. Nat. Chem. 2014, 6, No. 229.

(9) Bisoyi, H. K.; Li, Q. Light-Driven Liquid Crystalline Materials: from Photo-Induced Phase Transitions and Property Modulations to Applications. Chem. Rev. 2016, 116, 15089-15166.

(10) Koçer, G.; ter Schiphorst, J.; Hendrikx, M.; Kassa, H. G.; Leclère, P.; Schenning, A. P. H. J.; Jonkheijm, P. Light-Responsive Hierarchically Structured Liquid Crystal Polymer Networks for Harnessing Cell Adhesion and Migration. Adv. Mater. 2017, 29, No. 1606407.

(11) Gelebart, A. H.; Mulder, D. J.; Varga, M.; Konya, A.; Vantomme, G.; Meijer, E. W.; Selinger, R. L. B.; Broer, D. J. Making Waves in a Photoactive Polymer Film. Nature 2017, 546, 632-636.

(12) Pang, X.; Lv, J.-a.; Zhu, C.; Qin, L.; Yu, Y. Photodeformable Azobenzene-Containing Liquid Crystal Polymers and Soft Actuators. Adv. Mater. 2019, No. 1904224.

(13) Chen, M.; Yao, B.; Kappl, M.; Liu, S.; Yuan, J.; Berger, R.; Zhang, F.; Butt, H.-J.; Liu, Y.; Wu, S. Entangled AzobenzeneContaining Polymers with Photoinduced Reversible Solid-to-Liquid Transitions for Healable and Reprocessable Photoactuators. Adv. Funct. Mater. 2019, No. 1906752.

(14) Shahsavan, H.; Salili, S. M.; Jákli, A.; Zhao, B. Thermally Active Liquid Crystal Network Gripper Mimicking the Self-Peeling of Gecko Toe Pads. Adv. Mater. 2017, 29, No. 1604021.

(15) Babakhanova, G.; Turiv, T.; Guo, Y.; Hendrikx, M.; Wei, Q.-H.; Schenning, A. P. H. J.; Broer, D. J.; Lavrentovich, O. D. Liquid Crystal Elastomer Coatings with Programmed Response of Surface Profile. Nat. Commun. 2018, 9, No. 456. 
(16) Cao, A.; van Raak, R. J. H.; Pan, X.; Broer, D. J. Temperatureand Light-Regulated Gas Transport in a Liquid Crystal Polymer Network. Adv. Funct. Mater. 2019, 29, No. 1900857.

(17) Liu, Y.; Xu, B.; Sun, S.; Wei, J.; Wu, L.; Yu, Y. Humidity- and Photo-Induced Mechanical Actuation of Cross-linked Liquid Crystal Polymers. Adv. Mater. 2017, 29, No. 1604792.

(18) Wani, O. M.; Verpaalen, R.; Zeng, H.; Priimagi, A.; Schenning, A. P. H. J. An Artificial Nocturnal Flower via Humidity-Gated Photoactuation in Liquid Crystal Networks. Adv. Mater. 2019, 31, No. 1805985

(19) Lan, R.; Sun, J.; Shen, C.; Huang, R.; Zhang, L.; Yang, H. Reversibly and Irreversibly Humidity-Responsive Motion of Liquid Crystalline Network Gated by $\mathrm{SO}_{2}$ Gas. Adv. Funct. Mater. 2019, 29, No. 1900013.

(20) Stumpel, J. E.; Wouters, C.; Herzer, N.; Ziegler, J.; Broer, D. J.; Bastiaansen, C. W. M.; Schenning, A. P. H. J. An Optical Sensor for Volatile Amines Based on an Inkjet-Printed, Hydrogen-Bonded, Cholesteric Liquid Crystalline Film. Adv. Opt. Mater. 2014, 2, 459464.

(21) Lehmann, W.; Skupin, H.; Tolksdorf, C.; Gebhard, E.; Zentel, R.; Krüger, P.; Lösche, M.; Kremer, F. Giant Lateral Electrostriction in Ferroelectric Liquid-Crystalline Elastomers. Nature 2001, 410, $447-450$.

(22) Ohm, C.; Brehmer, M.; Zentel, R. Liquid Crystalline Elastomers as Actuators and Sensors. Adv. Mater. 2010, 22, 33663387.

(23) Agrawal, A.; Chen, H.; Kim, H.; Zhu, B.; Adetiba, O.; Miranda, A.; Chipara, A. C.; Ajayan, P. M.; Jacot, J. G.; Verduzco, R. Electromechanically Responsive Liquid Crystal Elastomer Nanocomposites for Active Cell Culture. ACS Macro Lett. 2016, 5, 13861390.

(24) Liu, D.; Tito, N. B.; Broer, D. J. Protruding Organic Surfaces Triggered by In-Plane Electric Fields. Nat. Commun. 2017, 8, No. 1526 .

(25) Feng, W.; Broer, D. J.; Liu, D. Oscillating Chiral-Nematic Fingerprints Wipe Away Dust. Adv. Mater. 2018, 30, No. 1704970.

(26) Feng, W.; Broer, D. J.; Liu, D. Combined Light and Electric Response of Topographic Liquid Crystal Network Surfaces. Adv. Funct. Mater. 2019, No. 1901681.

(27) Davidson, Z. S.; Shahsavan, H.; Aghakhani, A.; Guo, Y.; Hines, L.; Xia, Y.; Yang, S.; Sitti, M. Monolithic Shape-Programmable Dielectric Liquid Crystal Elastomer Actuators. Sci. Adv. 2019, 5, No. eaay0855.

(28) Liu, D.; Broer, D. J. Self-Assembled Dynamic 3D Fingerprints in Liquid-Crystal Coatings Towards Controllable Friction and Adhesion. Angew. Chem., Int. Ed. 2014, 53, 4542-4546.

(29) Liu, D.; Broer, D. J. Light Controlled Friction at a Liquid Crystal Polymer Coating with Switchable Patterning. Soft Matter 2014, 10, 7952-7958.

(30) Shahsavan, H.; Salili, S. M.; Jákli, A.; Zhao, B. Smart MuscleDriven Self-Cleaning of Biomimetic Microstructures from Liquid Crystal Elastomers. Adv. Mater. 2015, 27, 6828-6833.

(31) Gelebart, A. H.; Liu, D.; Mulder, D. J.; Leunissen, K. H. J.; van Gerven, J.; Schenning, A. P. H. J.; Broer, D. J. Photoresponsive Sponge-like Coating for On-Demand Liquid Release. Adv. Funct. Mater. 2018, 28, No. 1705942.

(32) Camargo, C. J.; Campanella, H.; Marshall, J. E.; Torras, N.; Zinoviev, K.; Terentjev, E. M.; Esteve, J. Localised Actuation in Composites Containing Carbon Nanotubes and Liquid Crystalline Elastomers. Macromol. Rapid Commun. 2011, 32, 1953-1959.

(33) Camargo, C. J.; Campanella, H.; Marshall, J. E.; Torras, N.; Zinoviev, K.; Terentjev, E. M.; Esteve, J. Batch Fabrication of Optical Actuators Using Nanotube-Elastomer Composites Towards Refreshable Braille Displays. J. Micromech. Microeng. 2012, 22, No. 075009.

(34) Torras, N.; Zinoviev, K.; Camargo, C. J.; Campo, E. M.; Campanella, H.; Esteve, J.; Marshall, J. E.; Terentjev, E. M.; Omastová, M.; Krupa, I.; Teplický, P.; Mamojka, B.; Bruns, P.; Roeder, B.; Vallribera, M.; Malet, R.; Zuffanelli, S.; Soler, V.; Roig, J.; Walker, N.; Wenn, D.; Vossen, F.; Crompvoets, F. M. H. Tactile
Device Based on Opto-Mechanical Actuation of Liquid Crystal Elastomers. Sens. Actuators, A 2014, 208, 104-112.

(35) Karageorgiev, P.; Neher, D.; Schulz, B.; Stiller, B.; Pietsch, U.; Giersig, M.; Brehmer, L. From Anisotropic Photo-Fluidity Towards Nanomanipulation in the Optical Near-Field. Nat. Mater. 2005, 4, 699-703.

(36) Fang, G. J.; Maclennan, J. E.; Yi, Y.; Glaser, M. A.; Farrow, M.; Korblova, E.; Walba, D. M.; Furtak, T. E.; Clark, N. A. Athermal Photofluidization of Glasses. Nat. Commun. 2013, 4, No. 1521.

(37) Hurduc, N.; Donose, B. C.; Macovei, A.; Paius, C.; Ibanescu, C.; Scutaru, D.; Hamel, M.; Branza-Nichita, N.; Rocha, L. Direct Observation of Athermal Photofluidisation in Azo-Polymer Films. Soft Matter 2014, 10, 4640-4647.

(38) Vapaavuori, J.; Laventure, A.; Bazuin, C. G.; Lebel, O.; Pellerin, C. Submolecular Plasticization Induced by Photons in Azobenzene Materials. J. Am. Chem. Soc. 2015, 137, 13510-13517.

(39) Zhou, H.; Xue, C.; Weis, P.; Suzuki, Y.; Huang, S.; Koynov, K.; Auernhammer, G. K.; Berger, R.; Butt, H.-J.; Wu, S. Photoswitching of Glass Transition Temperatures of Azobenzene-Containing Polymers Induces Reversible Solid-to-Liquid Transitions. Nat. Chem. 2017, 9, 145-151.

(40) Liu, D.; Broer, D. J. New Insights into Photoactivated Volume Generation Boost Surface Morphing in Liquid Crystal Coatings. Nat. Commun. 2015, 6, No. 8334.

(41) Kumar, K.; Schenning, A. P. H. J.; Broer, D. J.; Liu, D. Regulating the Modulus of a Chiral Liquid Crystal Polymer Network by Light. Soft Matter 2016, 12, 3196-3201.

(42) Kumar, K.; Knie, C.; Bléger, D.; Peletier, M. A.; Friedrich, H.; Hecht, S.; Broer, D. J.; Debije, M. G.; Schenning, A. P. A Chaotic SelfOscillating Sunlight-Driven Polymer Actuator. Nat. Commun. 2016, 7, No. 11975.

(43) Shimamura, A.; Priimagi, A.; Mamiya, J.-i.; Ikeda, T.; Yu, Y.; Barrett, C. J.; Shishido, A. Simultaneous Analysis of Optical and Mechanical Properties of Cross-linked Azobenzene-Containing Liquid-Crystalline Polymer Films. ACS Appl. Mater. Interfaces 2011, 3, 4190-4196.

(44) Yue, Y.; Norikane, Y.; Azumi, R.; Koyama, E. Light-Induced Mechanical Response in Crosslinked Liquid-Crystalline Polymers with Photoswitchable Glass Transition Temperatures. Nat. Commun. 2018, 9, No. 3234.

(45) Pilz da Cunha, M.; van Thoor, E.; Debije, M.; Broer, D. J.; Schenning, A. Unravelling the Photothermal and Photomechanical Contributions to Actuation of Azobenzene-Doped Liquid Crystal Polymers in Air and Water. J. Mater. Chem. C 2019, 7, 13502-13509.

(46) Yager, K. G.; Barrett, C. J. Photomechanical Surface Patterning in Azo-Polymer Materials. Macromolecules 2006, 39, 9320-9326.

(47) Harrison, J. M.; Goldbaum, D.; Corkery, T. C.; Barrett, C. J.; Chromik, R. R. Nanoindentation Studies to Separate Thermal and Optical Effects in Photo-Softening of Azo Polymers. J. Mater. Chem. C 2015, 3, 995-1003.

(48) van der Kooij, H. M.; Fokkink, R.; van der Gucht, J.; Sprakel, J. Quantitative Imaging of Heterogeneous Dynamics in Drying and Aging Paints. Sci. Rep. 2016, 6, No. 34383.

(49) van der Kooij, H. M.; Semerdzhiev, S. A.; Buijs, J.; Broer, D. J.; Liu, D.; Sprakel, J. Morphing of Liquid Crystal Surfaces by Emergent Collectivity. Nat. Commun. 2019, 10, No. 3501.

(50) Bolay, H.; Reuter, U.; Dunn, A. K.; Huang, Z.; Boas, D. A.; Moskowitz, M. A. Intrinsic Brain Activity Triggers Trigeminal Meningeal Afferents in a Migraine Model. Nat. Med. 2002, 8, 136142.

(51) Zakharov, P.; Völker, A. C.; Wyss, M. T.; Haiss, F.; Calcinaghi, N.; Zunzunegui, C.; Buck, A.; Scheffold, F.; Weber, B. Dynamic Laser Speckle Imaging of Cerebral Blood Flow. Opt. Express 2009, 17, 13904-13917.

(52) Nadort, A.; Kalkman, K.; Van Leeuwen, T. G.; Faber, D. J. Quantitative Blood Flow Velocity Imaging Using Laser Speckle Flowmetry. Sci. Rep. 2016, 6, No. 25258. 
(53) Heeman, W.; Steenbergen, W.; van Dam, G. M.; Boerma, E. C. Clinical Applications of Laser Speckle Contrast Imaging: a Review. J. Biomed. Opt. 2019, 24, No. 080901.

(54) Erpelding, M.; Amon, A.; Crassous, J. Diffusive Wave Spectroscopy Applied to the Spatially Resolved Deformation of a Solid. Phys. Rev. E 2008, 78, No. 046104.

(55) Amon, A.; Mikhailovskaya, A.; Crassous, J. Spatially Resolved Measurements of Micro-Deformations in Granular Materials Using Diffusing Wave Spectroscopy. Rev. Sci. Instrum. 2017, 88, No. 051804.

(56) Nagazi, M.-Y.; Brambilla, G.; Meunier, G.; Marguerès, P.; Périé, J.-N.; Cipelletti, L. Space-Resolved Diffusing Wave Spectroscopy Measurements of the Macroscopic Deformation and the Microscopic Dynamics in Tensile Strain Tests. Opt. Laser Eng. 2017, 88, 5-12.

(57) van der Kooij, H. M.; Susa, A.; García, S. J.; van der Zwaag, S.; Sprakel, J. Imaging the Molecular Motions of Autonomous Repair in a Self-Healing Polymer. Adv. Mater. 2017, 29, No. 1701017.

(58) van der Kooij, H. M.; Dussi, S.; van de Kerkhof, G. T.; Frijns, R. A. M.; van der Gucht, J.; Sprakel, J. Laser Speckle Strain Imaging Reveals the Origin of Delayed Fracture in a Soft Solid. Sci. Adv. 2018, 4, No. eaar1926.

(59) Verho, T.; Karppinen, P.; Gröschel, A. H.; Ikkala, O. Imaging Inelastic Fracture Processes in Biomimetic Nanocomposites and Nacre by Laser Speckle for Better Toughness. Adv. Sci. 2018, 5, No. 1700635.

(60) Buijs, J.; van der Gucht, J.; Sprakel, J. Fourier Transforms for Fast and Quantitative Laser Speckle Imaging. Sci. Rep. 2019, 9, No. 13279.

(61) Bonn, D.; Denn, M. M. Yield Stress Fluids Slowly Yield to Analysis. Science 2009, 324, 1401-1402.

(62) van Melick, H. G. H.; Govaert, L. E.; Meijer, H. E. H. On the Origin of Strain Hardening in Glassy Polymers. Polymer 2003, 44, 2493-2502.

(63) Corwin, E. I.; Jaeger, H. M.; Nagel, S. R. Structural Signature of Jamming in Granular Media. Nature 2005, 435, 1075-1078.

(64) Liu, D.; Bastiaansen, C. W. M.; den Toonder, J. M. J.; Broer, D. J. Light-Induced Formation of Dynamic and Permanent Surface Topologies in Chiral-Nematic Polymer Networks. Macromolecules 2012, 45, 8005-8012.

(65) Pine, D. J.; Weitz, D. A.; Zhu, J. X.; Herbolzheimer, E. Diffusing-Wave Spectroscopy: Dynamic Light Scattering in the Multiple Scattering Limit. J. Phys. Fr. 1990, 51, 2101-2127.

(66) Drobny, J. G. High Frequency (RF) Welding. In Handbook of Thermoplastic Elastomers; Elsevier, 2014; Chapter 4.12.2.6.

(67) Hollande, S.; Laurent, J.-L.; Lebey, T. High-Frequency Welding of an Industrial Thermoplastic Polyurethane Elastomer-Coated Fabric. Polymer 1998, 39, 5343-5349.

(68) Bisoyi, H. K.; Bunning, T. J.; Li, Q. Stimuli-Driven Control of the Helical Axis of Self-Organized Soft Helical Superstructures. Adv. Mater. 2018, 30, No. 1706512.

(69) Sprakel, J.; Zaccone, A.; Spaepen, F.; Schall, P.; Weitz, D. A. Direct Observation of Entropic Stabilization of bcc Crystals Near Melting. Phys. Rev. Lett. 2017, 118, No. 088003.

(70) Zaccone, A.; Scossa-Romano, E. Approximate Analytical Description of the Nonaffine Response of Amorphous Solids. Phys. Rev. B 2011, 83, No. 184205.

(71) Zaccone, A.; Terentjev, E. M. Disorder-Assisted Melting and the Glass Transition in Amorphous Solids. Phys. Rev. Lett. 2013, 110, No. 178002.

(72) Coussot, P.; Nguyen, Q. D.; Huynh, H. T.; Bonn, D. Avalanche Behavior in Yield Stress Fluids. Phys. Rev. Lett. 2002, 88, No. 175501.

(73) Printz, A. D.; Zaretski, A. V.; Savagatrup, S.; Chiang, A. S.-C.; Lipomi, D. J. Yield Point of Semiconducting Polymer Films on Stretchable Substrates Determined by Onset of Buckling. ACS Appl. Mater. Interfaces 2015, 7, 23257-23264.

(74) Dijksman, J. A.; Wortel, G. H.; van Dellen, L. T. H.; Dauchot, O.; van Hecke, M. Jamming, Yielding, and Rheology of Weakly Vibrated Granular Media. Phys. Rev. Lett. 2011, 107, No. 108303. 